\title{
REVIEW
}

\section{Coronary pressure measurement and fractional flow reserve}

\author{
N H J Pijls, B De Bruyne
}

Department of

Cardiology, Catharina Hospital, PO Box 1350, 5602 ZA Eindhoven,

Netherlands

N H J Pijls

Cardiovascular Center, Moorselbann 164, 9300

Aalst, Belgium

B De Bruyne

Correspondence to: Dr Pijls.

Accepted for publication 24 June 1998
The shortcomings of coronary arteriography to assess the physiological significance of coronary stenoses have been recognised for decades ${ }^{12}$; therefore, the importance of additional physiological techniques is beyond doubt. One of the currently available physiological techniques is coronary pressure measurement, which has emerged over the past few years as a major step forward in the invasive assessment of coronary artery disease..$^{3-9}$ This was partly owing to major technical progress in developing pressure monitoring guidewires and partly to a theoretical innovation, the concept of coronary pressure derived fractional flow reserve (FFR), which closely relates distal coronary pressure to myocardial blood flow during maximum arteriolar vasodilatation. In the catheterisation laboratory, this new approach enables an "on the spot" diagnosis as to

At maximum vasodilation
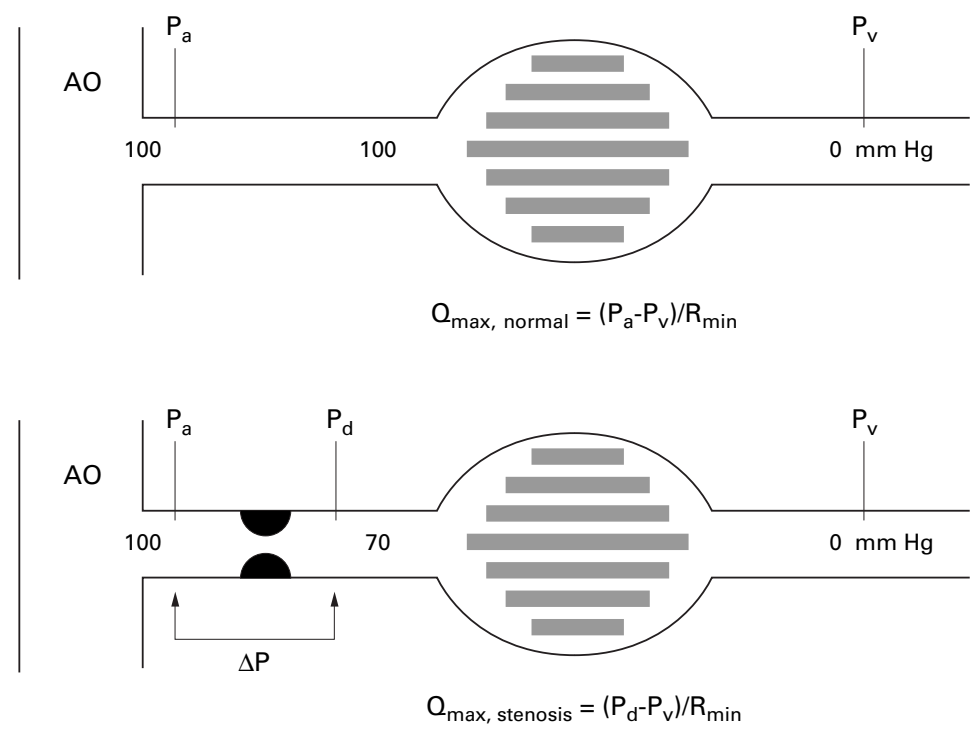

Figure 1 Schematic illustration of the coronary artery and its dependent myocardial vascular bed. Myocardial blood flow equals the perfusion pressure across the myocardium, divided by myocardial resistance. Because at maximum arteriolar vasodilatation resistance is minimal and constant $\left(R_{\text {min }}\right)$, maximum flow in the stenotic situation as a ratio to normal maximum flow equals the ratio of the myocardial perfusion pressure in the presence of the stenosis $\left(P_{d}-P_{v}\right)$ to normal myocardial perfusion pressure $\left(P_{a}-P_{v}\right)$, both measured after administration of a maximum hyperaemic stimulus. In other words, FFR equals $\left(P_{d}-P_{v}\right) /\left(P_{a}-P_{v}\right)$, which is generally very close to $P_{d} / P_{a}$. In this example, FFR equals 0.70 . AO, aorta; $P_{a}, P_{d}$, and $P_{v}$, mean aortic, distal coronary, and central venous pressure measured at maximum coronary hyperaemia; $Q_{\text {max , norme, }}$ maximum achievable myocardial flow if the coronary artery were normal; $Q_{\text {max } \text {,sensis }}$ maximum achievable myocardial flow in the presence of a stenosis; $R_{\min }$ indicates minimal resistance of the myocardial vascular bed. what extent a given epicardial stenosis contributes to reversible myocardial ischaemia and the decision whether revascularisation of the stenosis is warranted. In addition, pressure derived FFR appears to be a useful index to monitor and guide coronary intervention, particularly adequate stent deployment. ${ }^{10}{ }^{11}$

This review aims to provide a short overview of the theoretical and physiological background of this new approach and to focus on its clinical applicability, both for diagnostic and therapeutic catheterisation.

\section{Rationale of fractional flow reserve}

The functional state of a patient with a coronary artery stenosis is determined by the maximum blood flow that can reach the dependent myocardium. As soon as maximum achievable blood flow, at a given level of exercise, is no longer sufficient to match oxygen demand, myocardial ischaemia and angina pectoris will occur. Therefore, fundamentally, it is maximum blood flow that should be studied to establish the physiological significance of a coronary stenosis.

Fractional flow reserve is defined as maximum myocardial blood flow in the presence of a stenosis divided by the theoretical maximum flow in the absence of the stenosis - that is, maximum flow when the vessel is normal. This index represents that fraction of normal maximum flow that is still achievable despite the presence of the epicardial coronary stenosis. Therefore, it defines exactly to what extent a patient is limited by that stenosis.

Figure 1 show this concept and its calculation by coronary pressure measurement, with a simplified illustration of a coronary artery and the dependent myocardial bed. If that system is studied under conditions of maximum arteriolar vasodilatation, myocardial resistance will be minimal and equal to $\mathrm{R}_{\min }$. Under such circumstances the flow limiting effect of the epicardial stenosis is maximal, as is the pressure drop across the lesion.

As flow can be expressed as the ratio of driving pressure to resistance, maximum attainable blood flow through the myocardium equals the ratio of the perfusion pressure across the myocardium (distal coronary pressure minus central venous pressure) and the resistance $R_{\min }$. If there is no epicardial stenosis, the perfusion 

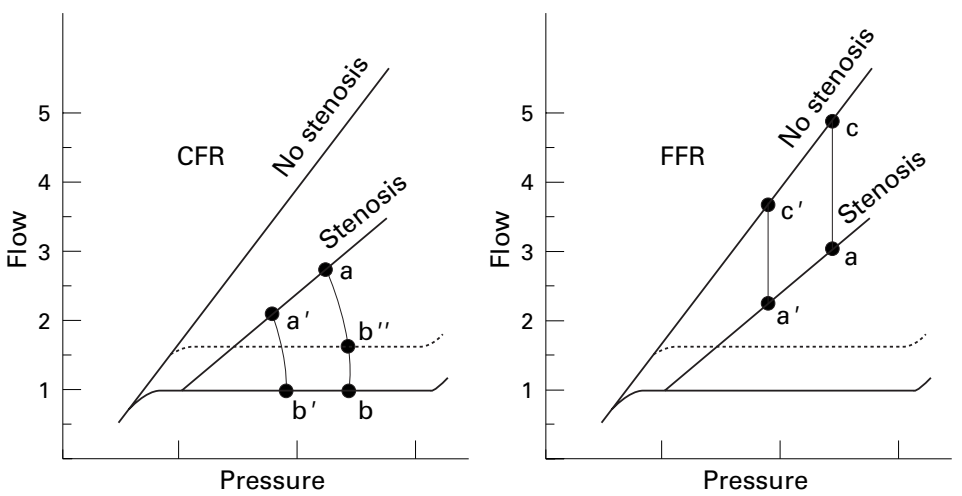

Figure 2 Features of coronary flow reserve (CFR) and fractional flow reserve (FFR). The coronary pressure-flow relation at maximum hyperaemia is indicated both for a normal coronary artery and a coronary stenosis. The resting pressure-flow relation (autoregulatory range) is represented by the solid horizontal line. (Left) CFR is defined as the ratio of hyperaemic to resting flow (a/b). It is obvious that with changing blood pressure, the value of $C F R$ changes to $a^{\prime} / b^{\prime}$. Also, when resting flow changes (as with changing heart rate or contractility) CFR changes to a/b". Therefore, no uniform normal value of CFR can be defined. (Right) FFR is defined as the ratio of maximum flow in the presence of a stenosis divided by normal maximum flow (a/c). This ratio remains unaffected by changes in blood pressure $\left(a / c=a^{\prime} / c^{\prime}\right)$ or by variations in resting flow. Its normal value is always 1.0 , irrespective of the patient, coronary artery, or prevailing haemodynamic conditions. Therefore, FFR is a more specific measure of functional stenosis severity.

pressure across the myocardium equals $P_{a}-P_{v}$, where $\mathrm{P}_{\mathrm{a}}$ is mean aortic pressure and $\mathrm{P}_{\mathrm{v}}$ is central venous pressure. In the presence of a coronary stenosis, the perfusion pressure across the myocardium has decreased to $P_{d}-P_{v}$, where $P_{d}$ represents hyperaemic coronary pressure distal to the stenosis.

Therefore, FFR is represented by:

$$
\mathrm{FFR}=\frac{\left(\mathrm{P}_{d}-\mathrm{P}_{v}\right) / \mathbf{R}_{\min }}{\left(\mathrm{P}_{a}-\mathrm{P}_{v}\right) / \mathbf{R}_{\min }}
$$

and because, at maximum coronary hyperaemia, resistance is minimal and constant, $R_{\min }$ cancels out and one obtains:

$$
\mathrm{FFR}=\frac{\left(\mathrm{P}_{d}-\mathrm{P}_{v}\right)}{\left(\mathrm{P}_{a}-\mathrm{P}_{v}\right)}
$$

If venous pressure $\left(P_{v}\right)$ is not increased as is usually the case, this is further simplified to:

$$
\mathrm{FFR}=\frac{\mathrm{P}_{d}}{\mathrm{P}_{a}}
$$

where $\mathrm{P}_{\mathrm{d}}$ and $\mathrm{P}_{\mathrm{a}}$ are simultaneously measured by a pressure guidewire and coronary catheter, respectively, after administration of a maximum vasodilating stimulus. As distal pressure $P_{d}$ is also affected by the extent of the collateral circulation, FFR also incorporates the effects of collaterals on myocardial perfusion. ${ }^{32}$ It is even possible to assess the separate contributions of coronary arterial and collateral blood flow to myocardial perfusion by performing coronary pressure measurements, ${ }^{3512}$ but that is beyond the scope of this short review.

\section{Features of FFR}

For practical decision making in the catheterisation laboratory, FFR has several advantages over classic coronary flow reserve (CFR) or coronary flow velocity reserve (CFVR) (fig 2). Whereas diminished CFR or CFVR cannot discriminate between epicardial disease, microvascular disease, or a combination of both,
FFR is a specific index for the epicardial stenosis and therefore better indicates to what degree a patient can be helped by revascularisation..$^{5-7}$

Secondly, FFR has a unique and unequivocal normal value of 1.0 in every patient and every coronary artery and, unlike CFR or CFVR, is completely independent of changes in heart rate, blood pressure, and contractility. ${ }^{513}$ As already mentioned, it takes into account the contribution of the collateral flow and, because there is no need for a normal reference artery, it can be applied in multivessel disease and for serial lesions within one vessel. ${ }^{35} 912$

It has been convincingly demonstrated that an FFR value $<0.75$ discriminates functionally significant lesions. ${ }^{561415}$ In a recent study, FFR more or less than 0.75 was compared directly to an ischaemic gold standard of all presently used non-invasive tests. ${ }^{6}$ Its diagnostic accuracy to predict inducible ischaemia correctly was approximately $95 \%$, and exceeded the diagnostic accuracy of thallium exercise testing and dobutamine stress echocardiography when performed as single tests. ${ }^{6}$

\section{Instrumentation to measure fractional \\ flow reserve}

With presently available equipment, pressure derived FFR can easily be determined at routine cardiac catheterisation with minimal risk and minimal prolongation of the procedure. The only necessary manipulations are crossing the interrogated stenosis with an 0.014 " pressure guidewire for reliable recording of trans-stenotic pressure, and administering a maximum hyperaemic stimulus. For the latter, intracoronary papaverine (8-12 mg), intracoronary adenosine (12 to $20 \mu \mathrm{g}$ ), or intravenous adenosine $(140 \mu \mathrm{g} / \mathrm{kg} / \mathrm{min}$ infused into the femoral vein) can be used. ${ }^{16}$

Recently, much progress has been made in the development and availability of pressure monitoring guidewires. In contrast to the older 0.018 " and 0.014 " fibreoptic wires, which were rather stiff and difficult to use in tortuous vessels, two new devices with excellent guidewire properties have been developed, either based on a high fidelity solid state pressure sensor or the fluid filled principle. The first is a disconnectable 0.014" electronic sensor tipped wire (PressureWire; Radi Medical Systems, Uppsala, Sweden), which is prepared and calibrated within seconds and has wire characteristics comparable to a regular high torque floppy guidewire. The second is a nitinol 0.014" fluid filled hollow wire (Informer; Scimed Inc, Maplegrove, Minnesota, USA) with an innovative superstiff connector at its proximal end, enabling recording of mean and phasic pressure recordings. Both wires can be used as first line guidewires throughout every interventional procedure, including exchanges of balloon and other catheters. Because the pressor sensor is located at a position $3 \mathrm{~cm}$ from the tip of the wires, it can safely be pulled back and pushed up across a stenosis without the need to cross the lesion again with the tip of the wire. Performing such a pull back curve 
during steady state maximum hyperaemia, as can be safely obtained using intravenous adenosine, is the most convincing and accurate demonstration of both the exact location and the severity of the stenosis. It is expected that more pressure guidewires will become available in the next few years.

For reliable measurement of coronary pressure, it is mandatory to use these ultrathin guidewires. When infusion catheters are used, even with a diameter of only $2.2 \mathrm{~F}$, significant and unpredictable overestimation of pressure gradient may occur, and erroneous results are obtained. ${ }^{1718}$

\section{Present clinical applications of coronary pressure measurement}

DIAGNOSTIC ANGIOGRAPHY

At present, the best established indication for coronary pressure measurement and FFR is as a diagnostic tool to determine whether a particular coronary stenosis, found at angiography, is responsible for reversible ischaemia (and consequently should be dilated or bypassed if medical treatment fails). The accuracy of FFR for that purpose, with a cut off point of 0.75 , is unsurpassed by any other invasive or non-invasive method. ${ }^{5-9}$

Specific applications in this respect are the intermediate stenosis, identification of the culprit lesion in case of multivessel disease, justification to perform (or avoid) angioplasty in a patient without documented evidence of ischaemia at non-invasive testing, and to indicate the exact location of a lesion in case of overprojection and other situations where the angiographic image is unclear. In all these cases, FFR $<0.75$ can be used as a justification to revascularise that lesion. ${ }^{5614}$ It has been shown retrospectively that it is safe to defer an intervention when FFR exceeds $0.75 .{ }^{6}{ }^{19}$ This position is presently being tested further in a large randomised prospective study (DEFER), which will be completed at the end of 1998 .

\section{CORONARY INTERVENTION}

It has been shown that a high value of FFR after regular balloon angioplasty is associated with a favourable long term outcome, and that there is a complementary value of FFR and angiography in evaluating angioplasty results. In a previous study, in patients with a post angioplasty FFR of $>0.90$, restenosis rates at 6,12 , and 24 months' follow up were $11 \%$, $12 \%$, and $15 \%$, respectively, compared with $29 \%, 32 \%$, and $42 \%$ in patients with a similar angiographic result but an FFR $<0.90 .{ }^{10}$ Further studies are warranted to confirm these data prospectively.

CORONARY STENTING

An important present application of coronary pressure measurement is the evaluation of optimum stent deployment. Optimum coronary stenting should at least be accompanied by normalisation of the conductance of the stented epicardial segment. Fractional flow reserve is a specific index of epicardial conductance, therefore, it is expected that FFR will be completely normal after adequate stent- ing or at least that, in the case of concomitant disease elsewhere in the artery, no noticeable hyperaemic pressure gradient will remain across a well deployed stent. Because the pressure sensor in the currently available wires is $3 \mathrm{~cm}$ from the tip, it is very easy to pull back and push the sensor repeatedly across the stent without having to cross the stent with the tip of the wire. In a recent study, 81 paired observations were made comparing both pressure measurements and intracoronary ultrasound (ICUS) side by side to evaluate optimal stent deployment. ${ }^{11}$ In that study, an almost perfect correlation was found between optimum stent deployment according to ICUS criteria and complete disappearance of the pressure gradient across the stent.

Therefore, coronary pressure measurements can be advocated as a cheap and rapid way to control adequate stent implantation if ICUS is not available. If there is still a pressure drop across the stent, ICUS remains necessary to detect the exact reason for that drop.

\section{Limitations of fractional flow reserve}

Most studies of FFR have been conducted in specific groups of patients with normal left ventricular function and without left ventricular hypertrophy. The value of coronary pressure measurement after myocardial infarction remains to be established. In left ventricular hypertrophy, the growth of the vascular bed is not proportional to the increase of myocardial muscle mass. As a result, the range of physiological reserve of maximum achievable blood flow corresponding with an FFR between 0.75 and 1 will become smaller with increasing severity of left ventricular hypertrophy; therefore, it is expected that the cut off value to indicate inducible ischaemia will be higher with increasing severity of hypertrophy. In such cases, an FFR of $>0.75$ cannot be used to rule out inducible ischaemia. Another limitation is exercise induced spasm, which will be missed because pharmacologically induced hyperaemia in the catheterisation laboratory in such patients is not comparable to exercise induced hyperaemia on the treadmill or bicycle. ${ }^{5}$ Finally, strictly speaking microvascular disease may influence FFR to some degree, because in such cases epicardial blood flow may not be as high as it could be without the microvascular disease and FFR might be overestimated. ${ }^{257}$ From a practical viewpoint, this last point is not a real limitation because coronary pressure measurements still indicate exactly to what extent the epicardial lesion contributes to the ischaemia and to what extent myocardial perfusion will be improved by intervention. As coronary pressure measurement is used more widely, more limitations and new applications will emerge.

Coronary pressure measurement provides the ability to obtain relevant physiological information in the catheterisation laboratory in an easy, cheap, rapid, and straightforward way. With the currently available pressure guidewires, excellent signals are obtained in each coronary artery within seconds, and timely decisions regarding revascularisation 
can be made. If angioplasty or stent implantation is performed, the same pressure wire can remain in place and be used as guidewire for the intervention, and to evaluate the results of the procedure without having to exchange wires therefore saving costs.

The secretarial assistance of Ingrid van de Kerkhof in preparing the manuscript is gratefully acknowledged.

1 Topol EJ, Nissen SE. Our preoccupation with coronary luminology: the dissociation between clinical and angiographic findings in ischemic heart disease. Circulation 1995;92:2333-42.

2 Kern JM, De Bruyne B, Pijls NHJ. From research to clinical practice: current role of intracoronary physiologically based decision making in the cardiac cathererization laboratory. $f$ Am Coll Cardiol 1997;30:613-20.

3 Pijls NHJ, van Son JAM, Kirkeeide RL, et al. Experimental basis of determining maximum coronary, myocardial, and collateral blood flow by pressure measurements for assessing functional stenosis severity before and after PTCA Circulation 1993;87:1354-67.

4 De Bruyne B, Baudhuin T, Melin JA, et al. Coronary flow reserve calculated from pressure measurements in humans. Validation with positron emission tomography. Circulation 1994;89:1013-22.

5 Piils NHJ, Van Gelder B, Van der Voort P, et al. Fractional flow reserve: a useful index to evaluate the influence of an epicardial coronary stenosis on myocardial blood flow. Circulation 1995;92:3183-93.

6 Pijls NHJ, De Bruyne B, Peels K, et al. Measurement of fractional flow reserve to assess the functional severity of coronary artery stenoses. N Engl f Med 1996;334:1703-8.

7 Wilson RF. Assessing the severity of coronary artery stenoses. N Engl F Med 1996;334:1735-7.

8 Lederman SJ, Menegus MA, Greenberg MA. Fractional flow reserve. ACC Current fournal Review 1997;2:34-35.
9 Pijls NHJ, De Bruyne B. Coronary pressure. Dortecht: Kluwer Academic Publishers, 1997.

10 Pijls NHJ, Bech GJW, De Bruyne B, et al. Prognostic value of pressure-derived fractional flow reserve to predict restenosis after regular ballon angioplasty [abstract]. Circulation 1997;96:I-649.

11 Hanekamp C, Koolen JJ, Pijls NHJ, et al. Comparison of quantitative coronary angiography, intravascular ultrasound, and pressure-derived fractional flow reserve to assess optimal stent deployment. Circulation. [In press.]

12 Pijls NHJ, Bech GJW, el Gamal MIH, et al. Quantification of recruitable coronary collateral blood flow in conscious recruitable coronary collateral blood flow in conscious humans and its potential to predict

13 De Bruyne B, Bartunek J, Sys SK, et al. Simultaneous coronary pressure and flow velocity measurements in humans. Circulation 1996;94:1842-9.

14 De Bruyne B, Bartunek J, Sys SU, et al. Relation between myocardial fractional flow reserve calculated from coronary pressure measurements and exercise-induced myocardial ischemia. Circulation 1995;92:39-46.

15 Bartunek J, Marwick Th, Rodriques ACT, et al. Dobutamine-induced wall motion abnormalities: correla-
tions with myocardial fractional flow reserve and quantitations with myocardial fractional flow reserve and quantitavive coro $1429-36$

16 Pijls NHJ, De Bruyne B, eds. Maximum hyperemic stimuli. Coronary pressure. Dortrecht: Kluwer Academic Publishers, 1997:96-104.

17 De Bruyne B, Sys SU, Heyndrickx GR. PTCA catheters versus fluid-filled pressure monitoring guidewires for coronary pressure measurements and correlation with quantitative coronary angiography. Am 7 Cardiol 1993;72: 1101-6

18 Pijls NHJ, De Bruyne B. Pitfalls in coronary pressure measurements. In: Coronary pressure. Dortrecht: Kluwer Academic Publishers, 1997:105-8.

19 Bech GJW, Pijls NHJ, De Bruyne B, et al. Long-term follow-up after deferral of PTCA of intermediate stenosis, based on coronary pressure measurement. F Am Coll Cardiol 1998;31:841-7. 